\title{
Ecoturismo y CONSERVAción: Perspectivas y PRÁcticas EN LAS RESERVAS DE LA BIÓSFERA DE LOS TUXTLAS, México y Maasai Mara, Kenia
}

\author{
Kennedy Obombo Magio \\ Programa Cátedras Conacyt \\ Instituto Tecnológico de Cancún \\ magiobom@yahoo.com·kmagio@conacyt.mx \\ Mónica Velarde Valadez \\ Universidad de Occidente \\ mvelardemx@yahoo.com·monica.velarde@udo.mx
}

\begin{abstract}
En este estudio se analiza la relación entre el ecoturismo y las actitudes de las comunidades locales hacia la conservación en dos reservas de la biósfera: Los Tuxtlas, México, y Maasai Mara, Kenia. La información se recopiló a través de una encuesta cuantitativa, entrevistas abiertas, cuatro grupos focales, así como la observación y participación directa. Se encontró que las actitudes y prácticas hacia la conservación se determinan por diferentes factores, entre ellos, beneficios directos e indirectos del ecoturismo, las costumbres, leyes y restricciones. También se advirtió que el ecoturismo puede generar impactos negativos como resultado de acciones involuntarias o equivocadas.
\end{abstract}

Palabras clave: Ecoturismo, conservación, desarrollo socioeconómico, reserva de la biósfera de Los Tuxtlas (México), reserva de la biósfera de Maasai Mara (Kenia).

\section{Ecotourism and conservation: Perspectives and practices in Los Tuxtlas Biosphere Reserve, Mexico y Maasai Mara Biosphere Reserve, Kenya}

The study presents an analysis of the relationship between ecotourism and conservation attitudes held by local community in two biosphere reserves: Los Tuxtlas, Mexico and Maasai Mara, Kenya. Information was collected through a quantitative survey administered by the researcher, open interviews, four focus group discussions, as well as direct observation and participation. It was found that conservation attitudes are determined by different factors, among them, direct and indirect benefits of ecotourism, customs, laws and restrictions. It was also found that ecotourism can generate negative impacts as a result of unintended or wrong actions.

Keywords: Ecotourism; Conservation; Socioeconomic Development; Los Tuxtlas Biosphere Reserve, Mexico; Maasai Mara Biosphere Reserve, Kenya.

Fecha de recepción: 8 de marzo de 2017. Fecha de aceptación: 7 de julio de 2017

CÓMO CITAR: Obombo, K. y Velarde, M. (2018). Ecoturismo y conservación: Perspectivas y prácticas en las reservas de la biósfera de Los Tuxtlas, México y Maasai Mara, Kenia. Dimensiones Turísticas, 2(2), 53-77. https://doi.org/10.47557/PMQQ9256 
- I surgimiento en todo el mundo de áreas naturales protegidas (ANP) es resultado de las políticas conservacionistas de los siglos xx y xxI (Toledo, 2005) y responde a un intento de la humanidad para proteger, conservar y mantener sitios de relevancia natural, cultural y ecológica. Según la Unión Internacional para la Conservación de la Naturaleza y los Recursos Naturales (International Union for the Conservation of Nature, 1994), son espacios terrestres o marinos dedicados a la protección y preservación de la diversidad biológica, y de los recursos naturales y culturales asociados con ella, y cuentan con instrumentos legales que definen su manejo. Se ha criticado la gestión tradicional de las ANP basada en la conservación estricta de la biodiversidad (Palomo et al., 2014), y muchas veces se limita la participación de las comunidades locales (véanse los trabajos de Hvenegaard, 1994; Vargas del Río y Brenner, 2013). Esto genera las siguientes preguntas fundamentales: ¿Para quién se crean estas unidades de conservación y a qué costo? Desdoblando este cuestionamiento, se señala que cualquier estilo de gestión ambiental que ignora la participación de las comunidades locales y les niega beneficios directos e indirectos, no contribuye a los principios de la sustentabilidad y está destinado a fracasar inevitablemente (Magio, Velarde, Santillán y Ríos, 2013).

Aún así, la gestión de las ANP ha evolucionado hacia el paradigma de uso múltiple en el cual se busca conciliar la conservación con el desarrollo local, reconociendo que la conservación es un acontecimiento social donde se interrelacionan diversas herramientas jurídicas, legales, jurisdiccionales, de ordenamiento territorial, educativas (Barriga, 2017). En este contexto, se destaca el concepto de reservas de la biósfera (RB), una subcategoría del grupo VI de las ANP planteada en el marco del Programa sobre el Hombre y la Biósfera (MAB, Man and the Biosphere Programme) de la Organización de las Naciones Unidas para la Educación, la Ciencia y la Cultura (Unesco) en 1970 y abarca una red mundial de paisajes representativos que se manejan como espacios de aprendizaje para el intercambio de información sobre conservación y desarrollo sustentable (Unesco, 1995). Este se ha convertido, dentro del paradigma del desarrollo sustentable, en uno de los conceptos dominantes en las políticas de conservación en el nivel internacional (Unesco, 2005).

Por su enfoque holístico, las RB han sido el escenario ideal para el establecimiento de actividades alternativas con el fin de disminuir la presión sobre los recursos naturales bajo el supuesto del desarrollo sustentable, las cuales incluyen turismo de naturaleza, ecoturismo, turismo de aventura, etc. (Barriga, 2015). El ecoturismo se ha reconocido como un instrumento tanto para conservar el patrimonio natural y cultural, como para promover un desarrollo sustentable. En diversos casos, se plantea como una alternativa económica que automáticamente cambia la manera en que los miembros de la comunidad local ven, perciben e interactúan con la biodiversidad, para apoyar las iniciativas de conservación (Honey, 2008). Este condicionamiento por enfoques y estímulos económicos produce fuertes impactos sociales, aumenta la inequidad, provoca trastornos ecológicos, entre otras cosas (Marín, García y Daltabuit, 2012). Por lo tanto, son relativamente raros los escenarios donde el desarrollo del ecoturismo en las áreas protegidas resulta tanto en la generación de bienestar económico y social como en la conservación ambiental (Agüera y Morales, 2015). 
CuAdro 1. Categorías analíticas (dimensiones e indicadores) del desarrollo ecoturístico sustentable en las áreas naturales protegidas

\begin{tabular}{|c|c|}
\hline DIMENSIONES & INDICADORES \\
\hline $\begin{array}{l}\text { 1. Estrategias } \\
\text { del desarrollo } \\
\text { ecoturístico }\end{array}$ & $\begin{array}{l}\text { i. ¿Quién controla las actividades de ecoturismo? } \\
\text { ii. La propiedad de las empresas/proyectos de ecoturismo (porcentajes de propiedad } \\
\text { local y de fuera). } \\
\text { iii. La distribución de los beneficios. ¿Qué porcentaje se queda en la comunidad? } \\
\text { iv. Son significativos estos beneficios para incentivar actitudes, perspectivas y } \\
\text { prácticas positivas hacia la conservación. } \\
\text { v. Gobernanza del ecoturismo: el papel del gobierno, las asociaciones locales de } \\
\text { ecoturismo. } \\
\text { vi. Política y planificación del ecoturismo. ¿Quién la desarrolla, quiénes participan? } \\
\text { vii. Participación y empoderamiento de la comunidad. } \\
\text { viii. ¿Quiénes gestionan los proyectos de ecoturismo? ¿Son personas de la comunidad } \\
\text { local o de fuera? } \\
\text { ix. ¿Cómo se realiza la actividad ecoturística? ¿Se realiza de manera que no perjudica } \\
\text { el bienestar de la reserva? }\end{array}$ \\
\hline 2. Conservación & $\begin{array}{l}\text { i. Actitudes y comportamientos de las comunidades hacia la conservación. } \\
\text { ii. Protección de los hábitats. } \\
\text { iii. Participación en la protección de las reservas. } \\
\text { iv. Restauración del hábitat (con fines ecoturísticos, iniciativas individuales o } \\
\text { colectivas de las comunidades locales, iniciativa pública o de las ONG Organización } \\
\text { no gubernamental). } \\
\text { v. Uso sustentable de los recursos naturales. } \\
\text { vi. Iniciativas de conservación privadas. } \\
\text { vii. Apoyo a la conservación de la flora, fauna y sus hábitats. } \\
\text { viii. Amenazas a la biodiversidad (como la caza de animales; comportamiento de los } \\
\text { ecoturistas). } \\
\text { ix. Concientización sobre conservación/educación ambiental (operadores, ecoturistas } \\
\text { y las comunidades locales). }\end{array}$ \\
\hline $\begin{array}{l}\text { 3. Desarrollo } \\
\text { socioeconómico de } \\
\text { las comunidades } \\
\text { locales }\end{array}$ & $\begin{array}{l}\text { i. Acceso al empleo. } \\
\text { ii. Nivel de ingresos. } \\
\text { iii. El acceso a la educación básica (primaria y secundaria). } \\
\text { iv. Sobrevivencia económica. } \\
\text { v. Estándares de la vida. } \\
\text { vi. Accesibilidad a la vivienda adecuada y digna. } \\
\text { vii. Estándares de salud. } \\
\text { viii. Acceso al crédito. } \\
\text { ix. Seguridad social y de empleo. } \\
\text { x. Beneficios indirectos del ecoturismo. }\end{array}$ \\
\hline
\end{tabular}
4. Factores que
i. Los factores legales (leyes y restricciones).
influyen en la
ii. Los factores económicos (la distribución de los costos y beneficios).
conservación
y desarrollo
iii. Los factores culturales (tradiciones y conductas culturales).
socioeconómico
iv. Los factores políticos (nivel de involucramiento y participación de las comunidades locales, vínculos y relación entre partes interesadas).
v. Los factores ecológicos (creación de las reservas).
vi. Los factores sociales (conductas sociales, características sociodemográficas).

Fuente: Elaboración propia, con base en Honey (2002), Picard (2015) y Weaver $(1998,2006)$. 
Ante esta situación, académicos y políticos están proponiendo investigaciones que mejoren la eficacia de las estrategias de conservación a través del desarrollo local (véase por ejemplo Honey, 2008; Eric et al., 2011; Brenner y Job, 2012; Vargas del Río y Brenner, 2013). Además, Guerrero Rodríguez (2010: 43) señala que es evidente "la falta de investigación acerca de las realidades existentes en los proyectos de ecoturismo", por lo que sugiere indagar sobre las diferentes estrategias empleadas en la implementación del concepto y evaluar si los proyectos mantienen una orientación hacia la conservación de los recursos naturales y si consideran la sustentabilidad social y cultural en los espacios donde se desarrolla. El cuadro 1 resume las categorías analíticas (dimensiones e indicadores) del desarrollo ecoturístico sustentable en las ANP, que se han utilizado en diversas investigaciones en este campo para determinar criterios de evaluación (Honey, 2002; Picard, 2015; Weaver, 1998, 2006).

Los objetivos fundamentales de la presente investigación fueron, por una lado, caracterizar el ecoturismo como una estrategia de conservación y, por el otro, analizar la relación entre los beneficios del ecoturismo y las actitudes de las comunidades involucradas hacia la conservación. Se plantearon tres hipótesis principales: 1. Los incentivos económicos por sí solos no son suficientes para transformar las actitudes y prácticas de las comunidades sobre la conservación; 2. Una mayor participación de las comunidades locales y la distribución equitativa de los costos y beneficios pueden generar actitudes y prácticas positivas que apoyen a la conservación; y 3. El ecoturismo puede influir negativamente en la conservación como resultado de acciones involuntarias o equivocadas.

\section{Fundamentos teóricos del eCoturismo, la CONSERVACión Y EL DESARROLLO SUSTENTABLE}

La sustentabilidad surgió durante la década de los ochenta como el enlace integral e inevitable entre el sistema natural y el desarrollo (Cardoso-Jiménez, 2006). El término fue utilizado por primera vez en 1987, dentro de un estudio realizado por la Comisión Mundial para el Medio Ambiente de la Organización de las Naciones Unidas, en donde, entre otras cosas, se llegó a la conclusión de que era necesario un cambio de fondo en el enfoque del desarrollo, ya que el planeta y todos sus sistemas ecológicos estaban sufriendo daños graves e irreversibles, debido a que los modelos tradicionales de desarrollo están basados en buena medida en visiones de corto plazo y de recursos ilimitados (Vargas del Río y Brenner, 2013). Pocos años después, se convertió en un término popular y de moda para la gestión de los recursos naturales (Gómez, 2011). De igual manera, el origen y la adopción del concepto turismo sustentable se ha vinculado con la evolución semántica del término desarrollo sustentable (Barriga, 2015). Adams y McShane (1992) afirman que la idea de integrar el turismo con la conservación probablemente ha existido desde los primeros días de safaris en África, y la creación de los parques nacionales en el siglo xIx. 
La relación entre turismo y sustentabilidad ofrece un panorama en el que aquel pudiera constituirse en una de las vías más eficaces para detonar procesos de desarrollo, enfatizando la participación de las poblaciones locales; específicamente, lo anterior se refiere al aprovechamiento de los recursos naturales y culturales por parte de las mencionadas poblaciones locales, mediante un turismo de corte alternativo (Laven, Wall-Reinius y Fredman, 2015).

En este tenor, Lawton y Weaver (2015) describen al turismo sustentable como "una forma humana de turismo a pequeña escala, que busca desarrollar y promover nuevas formas de turismo, las cuales generan beneficios para todos los participantes -viajeros, poblaciones locales y empresas turísticas- sin causar daño ecológico y social intolerable". El turismo sustentable, por lo tanto, responde al paradigma del desarrollo sustentable, buscando conciliar objetivos ambientales, económicos, sociales y culturales, mediante criterios como la planeación y gestión, la preservación ecológica, y la protección del patrimonio cultural y de la biodiversidad, para así lograr una producción sostenida a largo plazo y con equidad (Picard, 2015).

En el ámbito mundial, el turismo sustentable se ha ligado fuertemente con el diseño y manejo de ANP (Castillo, Baltazar, Lugo y Piñera, 2016). En especial, se destaca la creación de las RB, consideradas por Barriga (2017) como espacios de reconciliación entre conservación y desarrollo. Para cumplir con los objetivos de conservación, estas reservas se constituyen por zonas núcleo, donde se llevan a cabo actividades que están estrictamente vinculadas con la conservación, por ejemplo, educativas y de investigación; luego siguen las zonas tampón o de amortiguamiento, que por lo general rodean a las zonas núcleo, donde se establecen actividades compatibles, como educación ambiental, recreación, ecoturismo e investigación aplicada básica; y por último se encuentran las zonas de transición flexible, que comprenden variadas actividades (Primack y Ros, 2002).

Particularmente en las zonas de amortiguamiento y en las de transición flexible, las comunidades locales, los organismos de gestión, los científicos, las organizaciones no gubernamentales, los grupos culturales, el sector económico y otros interesados trabajan conjuntamente en la administración y el desarrollo sustentable del área (Unesco, 1995). En ese sentido, las principales funciones de las RB son: 1) conservación in situ, 2) desarrollo de áreas de demostración para uso sustentable y 3) apoyo logístico para los proyectos experimentales en investigación, monitoreo, educación ambiental y entrenamiento de las comunidades locales (Unesco-MAB, 1996 cit. en Barriga, 2017).

Se promueven modalidades sustentables del turismo en las ANP como alternativa de desarrollo por proveer beneficios ambientales, socioeconómicos y culturales (Castillo et al., 2016). El ecoturismo es una de las actividades económicas alternativas permitidas en la zona de amortiguamiento, ya que implica el desarrollo local con fines de conservar los recursos naturales; se considera esencialmente como un ejemplo de las estrategias de conservación contempladas en las RB. Más aún, una característica propia del ecoturismo es que, al ser una de las actividades alternativas, su desarrollo y aplicación no debe sustituir a aquellas que tradicionalmente realizan los pobladores de las comunidades en las que se realiza, sino constituir una actividad adicional o alternativa. 
Si bien se han documentado resultados satisfactorios derivados del turismo en ANP (como son los casos de Australia, Nueva Zelanda, Zanzíbar y Costa Rica), tanto en materia de conservación como en el empoderamiento de las comunidades y el fortalecimiento de la organización social (Barriga, 2015), la interacción de elementos ecológicos y sociales del turismo en áreas protegidas vuelve compleja su gestión y manejo; existen retos fundamentales que limitan la conciliación del desarrollo socioeconómico con la conservación de los recursos naturales. Por ejemplo, con frecuencia, los estudios han mostrado una desarticulación entre las acciones de las comunidades locales y las efectuadas por las instituciones gubernamentales (Castillo et al., 2016). Otro reto identificado es el menor peso asignado a la escala de la participación local; se ha revelado que solo un grupo selecto, por lo general las élites de la comunidad, participa o gestiona las actividades del desarrollo alternativo (Bello, Lovelock y Carr, 2017).

Estas cuestiones generan varias preguntas fundamentales, algunas de las cuales fueron respondidas en el presente estudio: ¿la participación de solo unas pocas personas garantiza beneficios para toda la comunidad? ¿Cómo influye la ecología política y la relación entre los actores en las iniciativas de conservación y desarrollo local? Si la comunidad no se beneficia, ¿es probable que cambien sus valores, creencias y prácticas de conservación? ¿Existen otros costos asociados con el ecoturismo (o cualquier otra actividad del desarrollo local compatible con la conservación) que las partes interesadas o los académicos ignoran? ¿Existen programas de distribución de costos y beneficios en empresas de ecoturismo basados en la comunidad, así como estrategias de gobernanza apropiadas que pueden contribuir de manera eficiente a la integración de conservación y desarrollo local? ¿Qué enfoques de gobernanza pueden reforzar la capacidad de organización local y mejorar las relaciones de las partes interesadas?

En general, cuando la participación de las comunidades locales es escasa y los beneficios mínimos, se originan actitudes negativas, como enojo, apatía y desconfianza de los pobladores, rechazo de las actividades de conservación y turismo, amenazas a la biodiversidad -por ejemplo, la caza furtiva-, conflicto entre seres humanos y vida silvestre, etc., lo que se convierte en un reto de gestión ambiental en las ANP. Al contrario, cuando las comunidades participan y perciben beneficios del turismo, emergen actitudes positivas: buena voluntad de los habitantes, apoyo a las actividades de conservación y turismo, custodia local de la biodiversidad, conservación de especies y hábitats en peligro de extinción, etcétera.

En el marco conceptual expuesto en la figura 1 se presenta una relación precisa de los factores que influyen en el turismo y las actitudes hacia la conservación en las ANP. Como se observa, las actividades turísticas en las ANP pueden generar dos tipos de actitudes hacia la conservación: positivas o negativas, dando lugar al éxito o al fracaso en su gestión.

En resumen, "lograr el éxito de integrar conservación y desarrollo socioeconómico a través del ecoturismo ha sido difícil” (Björk, 2007, p. 24), y los resultados varían de un caso a otro; esa valoración constituye una parte esencial del problema teórico y empírico de la presente investigación. Según Björk (2007, p. 24), existen diversos factores que influyen 


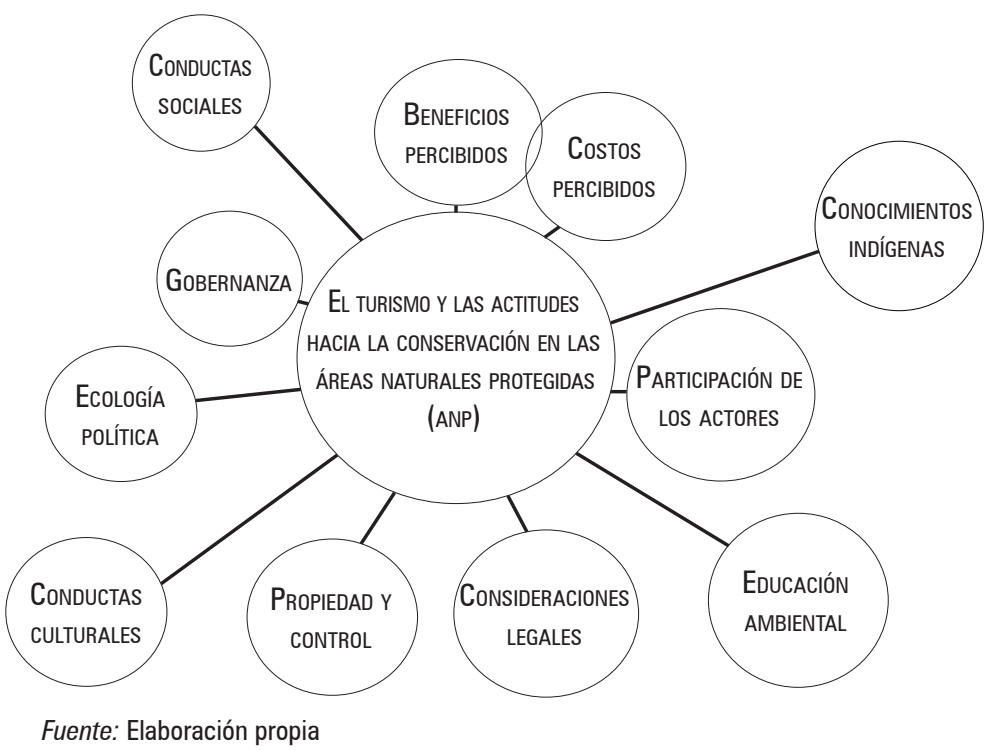

Figura 1. Marco conceptual de los factores que influyen en el turismo y las actitudes hacia la conservación en las Áreas Naturales Protegidas (ANP)

de manera significativa en el desempeño de los proyectos ecoturísticos en las RB, entre ellos, la escasa coordinación entre los actores, la propiedad de los negocios ecoturísticos, la participación local, la infraestructura existente, el estilo de gobernanza, así como las herramientas para gestionar el comportamiento de los turistas a fin de minimizar el impacto ecológico y fomentar la conservación (Brenner y Job, 2012).

\section{Metodología}

La investigación se desarrolló en cuatro grupos ejidales: los ejidos Adolfo López Mateos y Ruiz Cortines, ubicados en la reserva de la biósfera de Los Tuxtlas (RBLT), en el estado de Veracruz, México, y los ejidos Koiyaki (Koiyaki Group Ranch) y Lemek/Ol Chorro (Lemek Group Ranch), en la reserva de la biósfera de Maasai Mara (RBMM), en Kenia. Las cuatro comunidades se encuentran involucradas en las iniciativas de conservación y recientemente se han visto impactadas por proyectos ecoturísticos.

Es importante mencionar por qué se escogieron estos cuatro casos. Patton (1990) argumenta que no cualquier caso permite generar suficiente información para contestar las preguntas de investigación, por lo que se determinó un criterio en el cual se aseguró de que: 1. Existe el ecoturismo basado en la comunidad y que la comunidad participa en los proyectos ecoturísticos; 2. Los proyectos ecoturísticos incluidos en el estudio hayan sido establecidos hace al menos cinco años (el tiempo fue importante para evaluar los 
CuAdro 2. Criterios para la selección de los casos de estudio

\begin{tabular}{|c|c|c|c|c|}
\hline \multirow[b]{2}{*}{ CRITERIO de SELECcIón } & \multicolumn{2}{|c|}{ Casos de México } & \multicolumn{2}{|c|}{ Casos de Kenya } \\
\hline & $\begin{array}{c}\text { Ejido Adolfo López } \\
\text { Mateos }\end{array}$ & $\begin{array}{l}\text { Ejido Ruiz } \\
\text { Cortines }\end{array}$ & $\begin{array}{c}\text { Ejido Koiyaki } \\
\text { (Koiyaki Group } \\
\text { Ranch) }\end{array}$ & $\begin{array}{l}\text { Ejido Lemek/ Ol chorro } \\
\text { (Lemek Group Ranch) }\end{array}$ \\
\hline $\begin{array}{l}\text { Éxito reportado en la } \\
\text { literatura }\end{array}$ & Sí & Pocos datos & Sí & Sí \\
\hline $\begin{array}{l}\text { Influencia de centro } \\
\text { turístico masivo }\end{array}$ & Sí & Bastante & Sí & Sí \\
\hline Región & $\begin{array}{l}\text { Sierra de Santa } \\
\text { Marta al este del } \\
\text { Municipio de } \\
\text { Catemaco }\end{array}$ & $\begin{array}{l}\text { Sur del Volcán de } \\
\text { San Martín Tuxtla } \\
\text { en el municipio de } \\
\text { San Andrés Tuxtla }\end{array}$ & $\begin{array}{l}\text { Condado de Narok } \\
\text { Oeste }\end{array}$ & $\begin{array}{l}\text { Condado de Narok } \\
\text { Norte }\end{array}$ \\
\hline $\begin{array}{l}\text { Sistema de gestión } \\
\text { ambiental de } \\
\text { acuerdo con la Unión } \\
\text { Internacional para } \\
\text { la Conservación de } \\
\text { la Naturaleza y los } \\
\text { Recursos Naturales } \\
\text { (IUCN, por sus siglas } \\
\text { en inglés) }\end{array}$ & $\begin{array}{l}\text { Reserva de la } \\
\text { Biósfera-Los } \\
\text { Tuxtlas (RBLT) }\end{array}$ & $\begin{array}{l}\text { Reserva de la } \\
\text { Biósfera-Los } \\
\text { Tuxtlas (RBLT) }\end{array}$ & $\begin{array}{l}\text { Reserva de la } \\
\text { Biósfera-Maasai } \\
\text { Mara (RBMm) }\end{array}$ & $\begin{array}{l}\text { Reserva de la } \\
\text { Biósfera-Maasai } \\
\text { Mara (RBMm) }\end{array}$ \\
\hline $\begin{array}{l}\text { Existencia de } \\
\text { turismo basado en la } \\
\text { naturaleza }\end{array}$ & Sí & Sí & Sí & Sí \\
\hline $\begin{array}{l}\text { Alta participación } \\
\text { de las comunida- } \\
\text { des locales en las } \\
\text { actividades del } \\
\text { ecoturismo }\end{array}$ & Sí & Sí & Sí & Sí \\
\hline $\begin{array}{l}\text { Sistema de tenen- } \\
\text { cia, ambiental y } \\
\text { participación en } \\
\text { ecoturismo }\end{array}$ & Comunitario & Comunitario & Comunitario & Comunitario \\
\hline $\begin{array}{l}\text { Año de implemen- } \\
\text { tación formal del } \\
\text { proyecto de TBN a } \\
\text { nivel local }\end{array}$ & 1999 & 2008 & 1992 & 1996 \\
\hline $\begin{array}{l}\text { Número } \\
\text { de habitantes }\left({ }^{*}\right)\end{array}$ & $424(536)$ & $305(422)$ & $630(716)$ & $548(603)$ \\
\hline
\end{tabular}

${ }^{*}$ ) Estimación basada en el Instituto Nacional de Estadística Geográfica e Informática (INEGI, 2010) y Kenya Bureau of Statistics (KBS, 2012) - entre paréntesis, estimación después de los últimos censos. 
cambios en las prácticas y la distribución de beneficios); ${ }^{1} 3$. Que estos hayan sido desarrollados para fines de conservación, además de que incluyan los objetivos de desarrollo; 4. Que estén situados en o cerca de las RB; 5. Las comunidades involucradas demuestren la dependencia histórica de los recursos locales para sus necesidades de subsistencia; y 6. Se demuestre interés por parte de las comunidades y los actores de participar en la presente investigación.

Las seis consideraciones se investigaron por medio de un análisis de datos secundarios y una visita de exploración que se llevó a cabo durante noviembre de 2013 en Veracruz, México, y diciembre del mismo año en Maasai Mara, Kenia. Como se muestra en el cuadro 2, la investigación no solo se enfocó en la existencia de actividades turísticas basadas en la naturaleza, sino en la presencia de una amplia participación de la comunidad local en las actividades ecoturísticas o alguna estrategia de gestión que responde a los objetivos de conservación y desarrollo dentro del MAB, y que se considera como suficiente en la literatura académica (Honey, 2002, 2008; Higham, 2007; Adams y Hutton, 2007; Brenner y Job, 2012; Vargas del Río y Brenner, 2013).

Todos coinciden en un momento histórico, cuando el conflicto que surge de las necesidades de conservar o de utilizar los recursos naturales con fines turísticos es mediado por el discurso de la sustentabilidad, que busca incluir un cierto grado de participación social. Además, todas son localidades que poseen sistemas de manejo comunitario, y donde los habitantes han modificado en unos cuantos años su economía, pasando de la producción primaria y el autoconsumo, a una economía cada vez más enfocada en ofrecer servicios del turismo basado en la naturaleza y específicamente de ecoturismo. Las muestras se tomaron de las poblaciones que viven contiguas a las RB.

En el caso de la RBLT, se tomó como población meta cuatro municipios que rodean la reserva: San Andrés Tuxtla: 157364 habitantes y 39976 hogares; Catemaco: 48593 habitantes y 10470 hogares; Santiago Tuxtla: 56427 habitantes y 13840 hogares; y Hueyapan de Ocampo: 41649 habitantes y 9315 hogares; el total es de 304033 habitantes y 73601 hogares (Instituto Nacional de Estadística y Geografía, 2010). En cuanto a Kenia, se consideraron dos condados como población meta: Narok Oeste, con 196015 habitantes y 28002 hogares, y Narok Norte, con 242325 habitantes y 35617 hogares, cuyo total es de 438340 habitantes y 63619 hogares.

Del número total de hogares, se estima que solo 3900 y 2997 se encuentran contiguos a la RBLT y a la RBMM, respectivamente, y participan en actividades ecoturísticas. El tamaño de la muestra para la parte cuantitativa fue bastante grande (nRBLT=77 y nRB$\mathrm{MM}=69$ ) (véase cuadro 3). Este tamaño de muestra se calculó de acuerdo con la fórmula propuesta por Morse (2003), con una precisión de 5 \%, y 95 \% de nivel de confianza:

Donde: $\quad n=\frac{z^{2}(p \times q)}{e^{2}}$

1 Con base en el modelo de ciclos de vida de un destino (Butler, 1980), se puede argumentar que el ecoturismo en las dos reservas (la reserva de la biósfera de Los Tuxtlas y la reserva de la biósfera de Maasai Mara) se encuentra en la etapa de consolidación. 
$\mathrm{n}=$ tamaño de la muestra

$z=$ constante estadística que representa el nivel de confianza de $95 \%$ (1.96)

$p=$ proporción de éxito (3 900 $\div 33601$ para el caso de México, y $2997 \div 63619$ para el caso de Kenia); es decir, el número aproximado de los hogares involucrados de alguna manera en las actividades de ecoturismo dividido por el total de hogares en el área de estudio

$p=0.0530$ para el caso de la RBLT y 0.0471 para el caso de la RBMM

$q=$ proporción de fracaso $(1-p)=0.9470$ para el caso de la rblt y 0.9529 para el caso de la RBMM

e = error aceptable/precisión (5 \% o 0.05)

Se adoptó un enfoque de estudio de caso, lo que incluyó un examen profundo de las actividades de ecoturismo y conservación. La naturaleza del tema, los objetivos y la estrategia a través de la que se llevó a cabo esta investigación aglutinan componentes de la investigación aplicada, la evaluación sumativa y, en menor medida, la investigación básica. El componente de evaluación sumativa surgió en el esfuerzo por entender cómo el ecoturismo y sus supuestos beneficios influyen sobre las perspectivas y prácticas hacia la conservación. Por lo tanto, se examinó el grado en que el ecoturismo puede ser una intervención eficaz para estimular comportamientos relacionados con este concepto.

El diseño metodológico implicó la aplicación de métodos mixtos (cuantitativo y cualitativo), basándose en un paradigma interpretativo (Patton, 1990). Los métodos clave incluyeron una encuesta cuantitativa ( $n=138,95 \%$ de la muestra establecida), entrevistas abiertas ( $n=22,11$ en cada caso de estudio), cuatro grupos de enfoque, así como la participación y observación directa. Se aplicaron los instrumentos a los residentes locales y líderes de opinión en la comunidad. El cuestionario utilizado en la parte cuantitativa se estructuró de manera que respondiera al objetivo principal de la investigación: caracterizar el ecoturismo como una estrategia de conservación en las RB. En el diseño del instrumento (una escala de Likert), así como en la selección de los indicadores e ítems, se tuvieron en cuenta reflexiones de estudios previos sobre el tema (Vargas del Río y Brenner, 2013; Honey, 2008; Brenner y Job, 2012).

En las primeras dos secciones (A. Prácticas de conservación y B. Actitudes hacia la conservación) se incluyeron ítems (afirmaciones) sobre el impacto del ecoturismo y la conservación para detectar las actitudes de la comunidad local. En la última sección de la encuesta se buscó identificar los factores que influyen en el ecoturismo como estrategia de conservación. Es importante mencionar que la conceptualización del ecoturismo abarca una amplia variedad de dimensiones y criterios analíticos, por lo tanto, la selección de los indicadores e ítems incluidos en el análisis no es conclusiva. Sin embargo, permite dar un entendimiento global de los beneficios del ecoturismo y su influencia en las actitudes hacia la conservación.

El análisis cuantitativo fue descriptivo e inferencial, utilizando el paquete estadístico para ciencias sociales (sPSs versión 18). Para cada afirmación, los encuestados indicaron su nivel de concordancia (si estuvieron de acuerdo, en desacuerdo, o si el comentario les fue indiferente). A través de un análisis cuidadoso, se eligió una serie de afirmaciones 
CuAdro 3. El cálculo del tamaño de la muestra para la encuesta cuantitativa

\begin{tabular}{|c|c|c|c|c|c|c|}
\hline \multirow{3}{*}{$\begin{array}{l}\text { Caso de } \\
\text { estudio } \\
\text { Población } \\
\text { Meta } \\
\text { (Número de } \\
\text { habitantes en } \\
\text { los municipios } \\
\text { que abarcan } \\
\text { las Reservas } \\
\text { de la Biósfera) }\end{array}$} & \multirow{2}{*}{\multicolumn{4}{|c|}{ La reserva de la Biósfera de Los Tuxtlas (RBLT) }} & \multirow{2}{*}{\multicolumn{2}{|c|}{$\begin{array}{l}\text { La reserva de la Biósfera de Maasai } \\
\text { Mara (RBMM) } \\
\end{array}$}} \\
\hline & & & & & & \\
\hline & $\begin{array}{c}\text { San Andrés } \\
\text { Tuxtla }\end{array}$ & $\begin{array}{l}\text { Catemaco } \\
48593\end{array}$ & $\begin{array}{c}\text { de Santiago } \\
\quad 56427\end{array}$ & $\begin{array}{c}\text { Hueyapan } \\
\text { de Ocampo } \\
41649\end{array}$ & Narok Oeste & Narok Norte \\
\hline $\begin{array}{l}\text { Número de } \\
\text { hogares }\end{array}$ & 39976 & 10470 & 13840 & 9315 & 28002 & 35617 \\
\hline $\begin{array}{l}\text { Cálculo de la } \\
\text { muestra }\end{array}$ & \multicolumn{4}{|c|}{$\begin{array}{c}\mathrm{n}=\underline{\underline{z^{2}}(\mathrm{p} \times \mathrm{q})} \\
=\underline{\mathrm{e}^{2}} \\
=\frac{1.96}{\frac{(0.0530 \times(1-0.0530)}{0.05^{2}}} \\
=\frac{3.8416 \times(0.0530 \times 0.9470)}{0.0025} \\
\mathrm{n}_{\text {RBLT }}=\frac{0.1928137456}{0.0025} \\
\mathrm{n}_{\text {RBLT }}=77.1255 \\
\mathrm{n}_{\text {RBLT }}=77\end{array}$} & \multicolumn{2}{|c|}{$\begin{array}{c}\mathrm{n}=\underline{\underline{z}^{2}} \cdot(\mathrm{p} \times \mathrm{q}) \\
\mathrm{e}^{2} \\
\mathrm{n}_{\text {RBMM }}=\underline{1.96 \underline{2}} \frac{(0.0471 \times(1-0.0471)}{0.05^{2}} \\
\mathrm{n}_{\text {RBMM }}=\frac{3.8416 \times(0.0471 \times 0.9529)}{0.0025} \\
\mathrm{n}_{\text {RBMM }}=\underline{0.1724171161} \\
0.0025 \\
\mathrm{n}_{\text {RBMM }}=68.96685 \\
n_{\text {RBMM }}=69\end{array}$} \\
\hline $\begin{array}{l}\text { Número total } \\
\text { de habitantes } \\
\text { en cada grupo } \\
\text { ejidal }\left(^{*}\right)\end{array}$ & \multicolumn{2}{|c|}{ Ejido Adolfo López Mateos } & \multicolumn{2}{|c|}{ Ejido Ruiz Cortines } & $\begin{array}{l}\text { Ejido Koiyaki } \\
\text { (Koiyaki Group } \\
\text { Ranch) } \\
\quad 630(716)\end{array}$ & $\begin{array}{l}\text { Ejido Lemek/ 0l } \\
\text { Chorro (Lemek } \\
\text { Group Ranch) } \\
548 \text { (603) }\end{array}$ \\
\hline $\begin{array}{l}\text { Proporción } \\
\text { que repre- } \\
\text { senta de la } \\
\text { muestra }\end{array}$ & \multicolumn{2}{|c|}{$(424 \div 729 \times 100) 58 \%$} & \multicolumn{2}{|c|}{$(305 \div 729 \times 100) 42 \%$} & $\begin{array}{c}(630 \div 1178 \times 100) \\
53 \%\end{array}$ & $\begin{array}{c}(548 \div 1178 \times 100) \\
47 \%\end{array}$ \\
\hline $\begin{array}{l}\text { Número de } \\
\text { encuestas } \\
\text { previsto }\end{array}$ & \multicolumn{2}{|c|}{$\begin{array}{c}58 \% \text { de }\left(\mathrm{n}_{\text {RBLT }}\right)= \\
45\end{array}$} & \multicolumn{2}{|c|}{$\begin{array}{c}42 \% \text { de }\left(\mathrm{n}_{\text {RBLT }}\right)= \\
32\end{array}$} & $\begin{array}{c}53 \% \text { de }\left(\mathrm{n}_{\text {ввмM }}\right)= \\
37\end{array}$ & $\begin{array}{c}47 \% \text { de }\left(\mathrm{n}_{\text {RBMM }}\right)= \\
32\end{array}$ \\
\hline
\end{tabular}

$\left({ }^{*}\right)$ Se tomaron los datos oficiales de INEGI, 2010 y KBS, 2012 como base para el cálculo de la proporción de representación de la muestra

Fuente. Elaboración propia. 
que patentemente refleja las tendencias de conservación (actitudes y prácticas) en los cuatro grupos ejidales. Las afirmaciones escogidas para el análisis fueron las más claras y representativas, y se seleccionaron meticulosamente para reflejar las experiencias y conciencia general de los encuestados acerca de la relación entre ecoturismo y conservación. Además, se identificaron las afirmaciones cuyas respuestas difirieron de manera sustancial, donde la distribución de respuestas fue diferente de un grupo a otro; esta variación fue importante porque permitió el análisis estadístico adecuado (correlaciones) entre las variables dependientes (actitudes y prácticas de conservación) y las independientes (costos y beneficios del ecoturismo). Se utilizaron pruebas de Chi-cuadrado para determinar las asociaciones entre las actitudes hacia la conservación y posibles variables predictoras o explicativas, que en este caso fueron las variables independientes.

En cuanto a la parte cualitativa, se empleó la técnica llamada análisis de contenido o temático. Primero, se llevaron a cabo entrevistas que fueron grabadas, transcritas y analizadas junto con fuentes de información secundaria; también se realizaron otras entrevistas no grabadas y notas de campo mediante un proceso de asignación de códigos y recuperación de la información, mediado por una clasificación en categorías analíticas. Luego se organizaron las respuestas siguiendo los temas emergentes de los datos generados como resultado de las entrevistas. Como parte del análisis cualitativo, se identificaron patrones comunes de las respuestas y el desarrollo de temas que los reflejan. Para facilitar el análisis de la información cualitativa, se usó un programa de cómputo (ATLAS.ti) basado en la organización de la información mediante asignación de códigos y categorías de análisis. Se presentan citas textuales para apreciar mejor la variedad de las opiniones de los encuestados sobre los temas identificados. Por último, el estudio también incluyó una amplia revisión y análisis de documentos para complementar los datos primarios. Se utilizó una variedad de métodos de investigación y fuentes de datos para aumentar la triangulación, fortaleciendo de esta manera el diseño del estudio a través de controles de validez (véase Patton, 1990). Adicionalmente, mezclar herramientas cualitativas y cuantitativas en el presente estudio enriqueció la comprensión del trabajo final.

\section{Resultados de la investigación}

En la siguiente sección, se describen las actitudes y prácticas de las comunidades locales hacia la conservación, y las estrategias que aplican para usar y manejar los recursos naturales. Respondiendo a los dos objetivos de la investigación, esta sección proporciona una visión general de las actitudes y prácticas de conservación, que resume algunas similitudes y diferencias principales en las tendencias entre los cuatro grupos ejidales (se examina si el ecoturismo en las cuatro comunidades ha sido una alternativa económica/ herramienta/estrategia adecuada para incentivar actitudes y prácticas que favorecen la conservación en las reservas adyacentes). 


\subsection{Actitudes y prácticas de las comunidades hacia la conservación}

Uno de los principales hallazgos de los diferentes grupos ejidales es que los miembros de las comunidades consideran a la biodiversidad (flora y fauna) como parte importante de su sobrevivencia, por lo que reconocen la trascendencia de proteger los recursos naturales para las generaciones futuras. Sin embargo, ha sido difícil conciliar sus necesidades económicas con las de conservación. Respecto a los resultados del cuadro 4, se puede observar que miembros de la comunidad local en distintos grupos ejidales consideran ciertas prácticas como aceptables y otras no, dependiendo de la forma en que interpretan el concepto de conservación. El cuadro muestra una comparación de las actitudes hacia la conservación entre las cuatro comunidades, notándose que hay seis casos en los cuales las comunidades difieren de manera significativa en lo referente a las prácticas hacia la conservación. En general, los encuestados cuyas comunidades presentan actividad ecoturística disminuida (especialmente Ruiz Cortines y Lemek/OI Chorro) demostraron actitudes más negativas hacia la conservación, mientras que los de comunidades que experimentan mayor actividad ecoturística (Koiyaki y López Mateos) manifestaron actitudes más positivas (véase cuadro 4).

CuAdRo 4. Las actitudes y prácticas hacia la conservación en los cuatro grupos ejidales

\begin{tabular}{|c|c|c|c|c|c|c|}
\hline $\begin{array}{l}\text { Afirmaciones sobre } \\
\text { la conservación }\end{array}$ & $\begin{array}{c}\text { Nivel de } \\
\text { concordancia }\end{array}$ & $\begin{array}{l}\text { Ruiz Cortinez- } \\
\text { Tuxtlas (\%) }\end{array}$ & $\begin{array}{c}\text { Koiyaki- } \\
\text { Maasai } \\
(\%)\end{array}$ & $\begin{array}{c}\text { Adolfo López } \\
\text { Mateos- } \\
\text { Tuxtlas (\%) }\end{array}$ & $\begin{array}{c}\text { Lemek Ol } \\
\text { Chorro- } \\
\text { Maasai (\%) }\end{array}$ & $\begin{array}{l}\text { Nivel de } \\
\text { significan- } \\
\text { cia }\end{array}$ \\
\hline \multirow{2}{*}{$\begin{array}{l}\text { Más que para otra } \\
\text { cosa, los recursos } \\
\text { naturales (flora y } \\
\text { fauna) existen para } \\
\text { ser aprovechados } \\
\text { (leña, madera, } \\
\text { carne, etcétera). }\end{array}$} & De acuerdo & 22 & 12 & 28 & 47 & \multirow[t]{2}{*}{ * } \\
\hline & En desacuerdo & 76 & 82 & 64 & 45 & \\
\hline \multirow{2}{*}{$\begin{array}{l}\text { A veces es nece- } \\
\text { sario cortar árboles } \\
\text { para fines econó- } \\
\text { micos cuando uno } \\
\text { no tiene empleo. }\end{array}$} & De acuerdo & 30 & 46 & 58 & 74 & \multirow[b]{2}{*}{ * } \\
\hline & En desacuerdo & 54 & 42 & 34 & 21 & \\
\hline \multirow{2}{*}{$\begin{array}{l}\text { A veces es nece- } \\
\text { sariocazar anima- } \\
\text { les cuando uno no } \\
\text { tiene empleo. }\end{array}$} & De acuerdo & 14 & 26 & 52 & 44 & \multirow{2}{*}{ ** } \\
\hline & En desacuerdo & 80 & 69 & 46 & 55 & \\
\hline \multirow{2}{*}{$\begin{array}{l}\text { Debería ser } \\
\text { permitido para los } \\
\text { miembos de la } \\
\text { comunidad local } \\
\text { cortar árboles para } \\
\text { cultivar o practicar } \\
\text { la ganadería sin } \\
\text { ninguna restricción } \\
\text { de las autoridades. }\end{array}$} & De acuerdo & 22 & 2 & 17 & 31 & \multirow[t]{2}{*}{ * } \\
\hline & En desacuerdo & 54 & 86 & 81 & 57 & \\
\hline
\end{tabular}


CUADRo 4. Las actitudes y prácticas hacia la conservación en los cuatro grupos ejidales (finaliza)

\begin{tabular}{|c|c|c|c|c|c|c|}
\hline $\begin{array}{l}\text { Afirmaciones sobre } \\
\text { la conservación }\end{array}$ & $\begin{array}{c}\text { Nivel de } \\
\text { concordancia }\end{array}$ & $\begin{array}{l}\text { Ruiz Cortinez- } \\
\text { Tuxtlas (\%) }\end{array}$ & $\begin{array}{l}\text { Koiyaki- } \\
\text { Maasai } \\
(\%)\end{array}$ & $\begin{array}{c}\text { Adolfo López } \\
\text { Mateos- } \\
\text { Tuxtlas (\%) }\end{array}$ & $\begin{array}{l}\text { Lemek 이 } \\
\text { Chorro- } \\
\text { Maasai (\%) }\end{array}$ & $\begin{array}{l}\text { Nivel de } \\
\text { significan- } \\
\text { cia }\end{array}$ \\
\hline \multirow{2}{*}{$\begin{array}{l}\text { Si no fuera por la } \\
\text { conservación de } \\
\text { la reserva, habría } \\
\text { más oportunida- } \\
\text { des de generar } \\
\text { ingresos. }\end{array}$} & De acuerdo & 34 & 9 & 40 & 47 & \\
\hline & En desacuerdo & 57 & 76 & 58 & 41 & \\
\hline \multirow{2}{*}{$\begin{array}{l}\text { La caza de anima- } \\
\text { les silvestres se } \\
\text { considera buena } \\
\text { cuando uno ne- } \\
\text { cesita usarla para } \\
\text { generar ingresos. }\end{array}$} & De acuerdo & 2 & 16 & 11 & 23 & \\
\hline & En desacuerdo & 93 & 76 & 87 & 73 & \\
\hline \multirow{2}{*}{$\begin{array}{l}\text { Se involucraría } \\
\text { en prácticas de } \\
\text { uso intensivo de } \\
\text { recursos, en caso } \\
\text { de ganar más } \\
\text { dinero, sea de la } \\
\text { actividad turística } \\
\text { u otra actividad } \\
\text { económica. }\end{array}$} & De acuerdo & 73 & 56 & 75 & 99 & \\
\hline & En desacuerdo & 25 & 42 & 23 & 1 & $* * *$ \\
\hline \multirow{2}{*}{$\begin{array}{l}\text { El gobierno no } \\
\text { debe preocuparse } \\
\text { más por la con- } \\
\text { servación porque } \\
\text { los seres humanos } \\
\text { tienen más dere- } \\
\text { cho de vivir que los } \\
\text { animales. }\end{array}$} & De acuerdo & 34 & 42 & 28 & 31 & \\
\hline & En desacuerdo & 64 & 52 & 70 & 65 & \\
\hline \multirow{2}{*}{$\begin{array}{l}\text { Si la población de } \\
\text { animales fuera } \\
\text { alta, estaría bien } \\
\text { la caza. }\end{array}$} & De acuerdo & 38 & 42 & 40 & 71 & \multirow{2}{*}{ ** } \\
\hline & En desacuerdo & 57 & 56 & 46 & 27 & \\
\hline \multirow{2}{*}{$\begin{array}{l}\text { No es bueno cazar } \\
\text { animales salvajes. }\end{array}$} & De acuerdo & 67 & 59 & 70 & 73 & \\
\hline & En desacuerdo & 18 & 26 & 23 & 21 & \\
\hline \multirow{2}{*}{$\begin{array}{l}\text { Los bosques exis- } \\
\text { ten únicamente } \\
\text { para ser aprove- } \\
\text { chados económi- } \\
\text { camente }\end{array}$} & De acuerdo & 35 & 12 & 28 & 44 & \\
\hline & En desacuerdo & 58 & 79 & 64 & 51 & \\
\hline
\end{tabular}


En el cuadro 4 se encontraron seis respuestas que difirieron significativamente. Para determinar las relaciones específicas que contribuyeron a las significativas diferencias entre las comunidades, se realizaron pruebas de Chi-cuadrado comparando dos comunidades a la vez (véase cuadro 5). La primera línea de cada afirmación se refiere a la prueba entre las dos comunidades cuyas diferencias fueron las más claras.

Cuadro 5: La significación estadística de las asociaciones en el cuadro 4

\begin{tabular}{|c|c|c|c|c|c|c|}
\hline $\begin{array}{l}\text { Afirmaciones que muestran } \\
\text { asociaciones (diferencias) } \\
\text { significativas entre las comu- } \\
\text { nidades }\end{array}$ & $\begin{array}{l}\text { Grupo } \\
\text { ejidal }\end{array}$ & $\begin{array}{l}\text { En desacuerdo } \\
(\%)\end{array}$ & $\begin{array}{l}\text { Grupo } \\
\text { ejidal }\end{array}$ & $\begin{array}{c}\text { En desacuerdo } \\
(\%)\end{array}$ & $\begin{array}{c}\text { Valores } \\
\mathrm{p}\end{array}$ & $\begin{array}{l}\text { Niveles de } \\
\text { significancia }\end{array}$ \\
\hline \multirow{4}{*}{$\begin{array}{l}\text { Más que para otra cosa, los } \\
\text { recursos recursos naturales } \\
\text { (flora y fauna)existenpara ser } \\
\text { aprovechados (leña, madera, } \\
\text { carne, etcétera). }\end{array}$} & $\mathrm{KY}$ & 82 & LOC & 45 & 0.0409 & $*$ \\
\hline & $\mathrm{KY}$ & 82 & ALM & 64 & 0.0829 & \\
\hline & $\mathrm{RC}$ & 76 & LOC & 45 & 0.0307 & $*$ \\
\hline & ALM & 64 & LOC & 45 & 0.7772 & \\
\hline \multirow{3}{*}{$\begin{array}{l}\text { A veces es necesario cortar } \\
\text { árboles para fines económicos } \\
\text { cuando uno no tiene empleo. }\end{array}$} & $\mathrm{RC}$ & 54 & LOC & 21 & 0.0241 & * \\
\hline & $\mathrm{KY}$ & 42 & LOC & 21 & 0.0421 & * \\
\hline & $\mathrm{RC}$ & 54 & ALM & 34 & 0.1955 & \\
\hline \multirow{3}{*}{$\begin{array}{l}\text { A veces es necesario cazar } \\
\text { animales cuando uno no tiene } \\
\text { empleo. }\end{array}$} & $\mathrm{RC}$ & 80 & ALM & 46 & 0.0038 & $* *$ \\
\hline & $\mathrm{RC}$ & 80 & LOC & 55 & 0.0011 & $* *$ \\
\hline & $\mathrm{KY}$ & 69 & ALM & 46 & 0.1674 & \\
\hline \multirow{4}{*}{$\begin{array}{l}\text { Debería ser permitido para los } \\
\text { miembros de la comunidad } \\
\text { local cortar árboles para cul- } \\
\text { tivar o practicar la ganadería } \\
\text { sin ninguna restricción de las } \\
\text { autoridades. }\end{array}$} & $\mathrm{KY}$ & 86 & $\mathrm{RC}$ & 54 & 0.0110 & * \\
\hline & $\mathrm{KY}$ & 86 & LOC & 57 & 0.0251 & * \\
\hline & $\mathrm{KY}$ & 86 & ALM & 81 & 0.3422 & \\
\hline & ALM & 81 & LOC & 57 & 0.1510 & \\
\hline \multirow{4}{*}{$\begin{array}{l}\text { Se involucraría en prácticas de } \\
\text { uso intensivo de recursos, en } \\
\text { caso de ganar más dinero, sea } \\
\text { de la actividad turística u otra } \\
\text { actividad económica }\end{array}$} & $\mathrm{KY}$ & 56 & LOC & 99 & 0.0001 & $* * *$ \\
\hline & $\mathrm{RC}$ & 73 & LOC & 99 & 0.0004 & $* * *$ \\
\hline & ALM & 76 & LOC & 99 & 0.0011 & $* *$ \\
\hline & $\mathrm{KY}$ & 56 & ALM & 76 & 0.3481 & \\
\hline \multirow{3}{*}{$\begin{array}{l}\text { Si la población de animales } \\
\text { fuera alta, estaría bien la caza. }\end{array}$} & $\mathrm{RC}$ & 57 & LOC & 27 & 0.0035 & $* *$ \\
\hline & ALM & 46 & LOC & 27 & 0.0206 & * \\
\hline & $\mathrm{KY}$ & 56 & LOC & 27 & 0.0216 & * \\
\hline
\end{tabular}

$\mathrm{RC}=$ Ejido Ruiz Cortines- Tuxtlas; ALM = Ejido Adolfo López Mateos- Tuxtlas; $\mathrm{KY}=$ Ejido Koiyaki- Maasai Mara; LOC = Ejido Lemek OI Chorro.

Los niveles de significación se representan de la manera siguiente: "**" se refiere a $p<.05$, "*" se refiere a $\mathrm{p}<.01 \mathrm{y}$ "***" se refiere a $\mathrm{p}<.001$.

Fuente. Elaboración propia. 
Con base en los resultados de los cuadros 4 y 5 , se demuestra que las actitudes y prácticas de conservación se determinan por diferentes factores: oportunidades del ecoturismo, situación económica de los residentes, costumbres y circunstancias de diferentes comunidades. Por ejemplo, la comunidad de Lemek/Ol Chorro tuvo el mayor porcentaje (75\%) de las personas que consideran que "a veces es necesario cortar árboles para fines económicos cuando uno no tiene empleo". Los miembros de este grupo ejidal no están involucrados activamente en las actividades de ecoturismo como en otras comunidades, las tasas de desempleo son bastante altas en la zona y los miembros de la comunidad dependen en gran medida de la madera y la ganadería. Al contrario, la comunidad de Ruiz Cortines, que está más involucrada en actividades ecoturísticas y no depende de la madera, generó el porcentaje más bajo $(31$ \%) de las personas que están de acuerdo con la afirmación anterior (la diferencia en las respuestas de las dos comunidades fue estadísticamente significativa en $\mathrm{p}<0.05$ ).

Los encuestados de la comunidad Koiyaki, donde el ecoturismo es dominante, estuvieron en desacuerdo en que "debería ser permitido para los miembros de la comunidad local cortar árboles para cultivar o practicar la ganadería sin ninguna restricción de las autoridades", mientras que comunidades como Lemek/OI Chorro están a favor de la tala de árboles sin restricciones por parte del gobierno. Estas actitudes negativas pueden deberse a su dependencia de la agricultura y la ganadería. Además, han tenido experiencias negativas (conflictos) en el pasado con las autoridades del gobierno y gestores de la reserva en cuanto a las restricciones (la diferencia en las respuestas de Koiyaki y Lemek/OI Chorro fueron estadísticamente significativas en $p<0.05$ ). Asimismo, se destaca la variación en las prácticas de los residentes y su impacto sobre el medio ambiente. Por ejemplo, en Ruiz Cortines, 89 \% de los encuestados está de acuerdo en lo referente a los controles de la cacería, pero solo 28 \% está de acuerdo en cuanto a los controles relacionados con la escala de agricultura. Uno de los entrevistados comentó: "Los gestores de la reserva no entienden que ganamos la vida principalmente a través de la agricultura (...) el ecoturismo no es suficiente para todos nosotros (...) y los que ganan del ecoturismo, el ingreso no les alcanza para nada".

En términos generales, los encuestados de los cuatro grupos fueron casi unánimes en dos aspectos: primero, es de gran importancia que las generaciones futuras tengan la posibilidad de disfrutar de los bosques y los animales silvestres, y segundo, que los árboles clasificados entre las especies en peligro de extinción nunca se deberían de cortar. Estos resultados muestran que los miembros de las comunidades locales adyacentes a áreas protegidas por lo menos reconocen el delicado vínculo entre la conservación y las necesidades de las generaciones futuras.

Se buscó saber si había disminuido la tala de árboles y la cacería en los diferentes grupos ejidales y cuáles fueron las causas. En general, los encuestados estuvieron de acuerdo en que la tala de árboles y la caza de animales había disminuido (79 y $90 \%$ de los encuestados, respectivamente). El resultado más sorprendente es que lo atribuyen principalmente a las leyes y restricciones impuestas por los gestores de la reserva y no tanto al desarrollo del ecoturismo. Aún así, estuvieron de acuerdo en que tanto el ecoturismo como 
el aumento de la conciencia ambiental han tenido un impacto significativo (aunque sea mínimo) en el cambio de las actitudes y prácticas de conservación.

Estos resultados coinciden con lo que encontraron Siewe, Vadjunec y Caniglia (2017) y Ghimire (1994): que la gestión de las áreas protegidas a través de restricciones estrictas ha logrado disminuir la cacería y la deforestación. Durante las entrevistas, se identificaron fuertes sentimientos de animosidad entre los miembros de la comunidad y los gestores de las reservas; los encuestados atribuyen tales conflictos a la aplicación estricta de las normas y reglamentos dentro y fuera de las reservas. Sin embargo, esta consideración no debe ser malinterpretada; no se trata de defender el uso ilimitado de los recursos naturales, sino un cambio de la estrategia que busca influenciar las actitudes y percepciones de las comunidades locales a través de incentivos, más que el uso de la fuerza.

\subsection{Los impactos del ecoturismo y la conservación}

Con referencia a los hallazgos, queda claro que las prácticas de conservación en las cuatro comunidades tienen una fuerte asociación con el empleo del ecoturismo; por ejemplo, se manifestó que las familias que cuentan con empleo o las que participan en la actividad ecoturística tienen el mayor porcentaje de tierras con pastos en recuperación (80 \%) o en reforestación (75\%). El análisis determinó una asociación estadísticamente significativa $(p<0.001)$ entre participación o beneficios del ecoturismo y las prácticas de conservación. Sin embargo, se localizó una relación menos clara entre empleo, exposición y participación en la actividad ecoturística y las actitudes hacia la conservación.

Se encontraron asociaciones en únicamente cinco de las 12 correlaciones realizadas (pruebas de Chi-cuadrado) entre empleo o participación y las actitudes hacia la conservación, y solo una de las cinco asociaciones fue estadísticamente significativa en $p<.05$ (los encuestados que cuentan con empleo en comercios ecoturísticos y los que no); la afirmación de conservación en donde difirieron fue: “debería ser permitido para los miembros de la comunidad local cortar árboles para cultivar o practicar la ganadería sin ninguna restricción de las autoridades".

Por otro lado, hay varias afirmaciones que muestran tendencias inesperadas en cuanto a las actitudes hacia la conservación. Por ejemplo, se esperaba que los residentes beneficiados del ecoturismo mostraran el mayor porcentaje de respuestas positivas (demostrando actitudes positivas para la conservación) en comparación con los que generan pocos beneficios; sin embargo, es sorprendente que los encuestados del ejido Ruiz Cortinez, cuya participación y beneficios de las actividades ecoturísticas son mínimos, exhiben el mayor porcentaje de respuesta en desacuerdo con la afirmación "a veces es necesario cazar animales cuando uno no tiene empleo". Tomando la afirmación anterior como variable dependiente, la prueba Chi-cuadrado generó una asociación estadísticamente significativa en $p<0.001$ al comparar la respuesta de Ruiz Cortinez y Koiyaki.

No está claro por qué los encuestados de Ruiz Cortinez, una comunidad que recibe beneficios mínimos, dieron respuestas más opuestas a la cacería que los residentes de 
Koiyaki, un centro ecoturístico más establecido y que evidentemente produce mayor derrama económica que el anterior. Una de las razones puede ser la escasez general de animales salvajes en la RBLT, así como el aumento de la conciencia ambiental en la comunidad, y especialmente entre los líderes del grupo ejidal.

Aunque el empleo de la familia como un predictor de actitudes manifestó una tendencia inesperada, otras variables predictoras (como niveles de responsabilidad en la actividad ecoturística, ingresos indirectos, interacción e intercambio de ideas con ecoturistas, así como mejoras en la infraestructura) revelaron tendencias orientadas a la conservación (así se esperaba). Sin embargo, de todas las variables predictoras antes mencionadas, se halló significancia estadística únicamente en dos de las respuestas: intercambio de ideas con turistas y mejoras en la infraestructura.

Con base en las pruebas de Chi-cuadrado, se señala que la asociación entre el empleo y las actitudes hacia la conservación no mostró una tendencia clara (a pesar de que esta misma relación es más evidente en los hallazgos de las entrevistas); de hecho, las variables vinculadas con el beneficio indirecto del ecoturismo -por ejemplo, mejoras en la infraestructura e intercambio de ideas con turistas- expresaron una relación más fuerte y clara con las actitudes hacia la conservación que el empleo.

Hubo otras sorpresas similares; por ejemplo, la asociación entre niveles de responsabilidad en la actividad ecoturística y las actitudes hacia la conservación: se esperaba que los encuestados cuyos familiares tenían niveles más altos de responsabilidad en el ecoturismo manifestaran actitudes positivas, orientadas a la conservación, en comparación con los que manejaban bajos niveles de responsabilidad; empero, tal escenario fue evidente únicamente en seis de las 12 afirmaciones sobre la conservación, además, solo un caso de las seis asociaciones fue estadísticamente significativo.

A pesar de que los resultados no demuestran una fuerte asociación entre responsabilidad en la actividad ecoturística y actitudes hacia la conservación, no se descarta esta posibilidad; se aclara que sí existe una relación positiva entre diferentes niveles de responsabilidad y las actitudes hacia la conservación; se enfatiza también que todos los otros beneficios del ecoturismo, salvo el empleo directo, mostraron una asociación positiva como predictoras de las actitudes hacia la conservación; sin embargo, tales asociaciones no fueron claras o estadísticamente significativas.

También se encontró que la magnitud en la cual se opera el ecoturismo genera diferentes niveles de beneficios e impactos (cuando la escala es grande, se producen más beneficios y viceversa). Honey (2008) obtuvo resultados semejantes cuando realizó un estudio comparativo que abarcó casos de dos países para examinar los beneficios e inconvenientes del ecoturismo.

En Lemek/OI Chorro y Ruiz Cortines, donde el ecoturismo ocupa un papel secundario en la economía local, se encontraron pocos residentes que generan beneficios de la actividad ecoturística. Asimismo, la mayoría no experimenta efectos negativos del ecoturismo (de hecho, algunos de los encuestados quedaron sorprendidos cuando se les preguntó sobre 


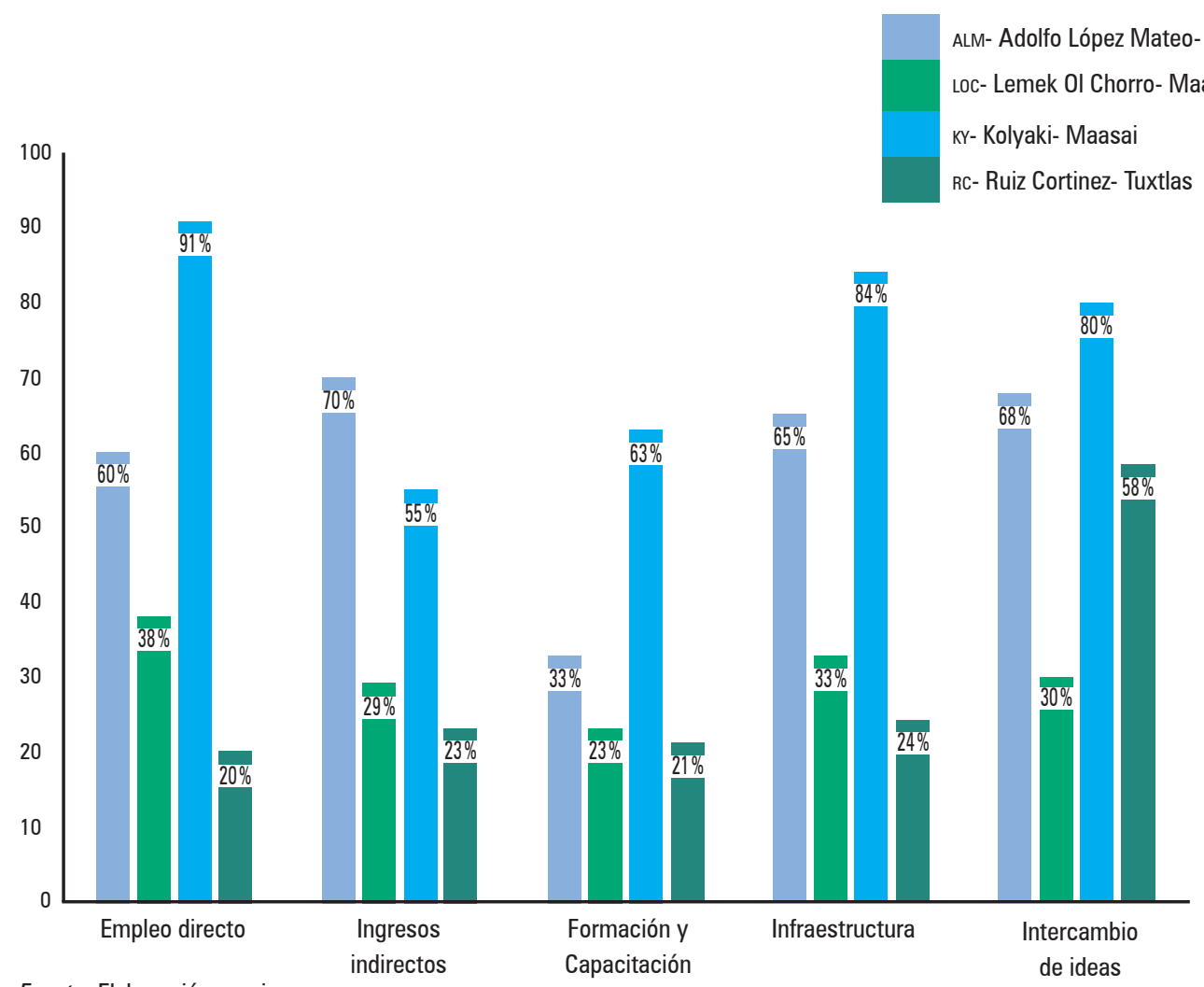

Fuente: Elaboración propia.

FiguRA 2. Distribución de beneficios en los cuatro grupos ejidales

el impacto del ecoturismo). Al contrario, las economías locales de Koiyaki y Adolfo López Mateos dependen en gran medida del ecoturismo.

Como se observa en las figuras 2 y 3 , estas dos comunidades se han beneficiado más del ecoturismo que Lemek/OI Chorro y Ruiz Cortines. De igual manera, se han generado más impactos (socioculturales, económicos y ambientales) en esas comunidades, como resultado de una mayor actividad ecoturística.

Aunque la mayoría de esos impactos se refieren específicamente al ecoturismo, cabe reconocer que representan una amenaza potencial al desarrollo económico alternativo en las comunidades y, por consecuencia, al bienestar de los residentes a largo plazo. Un buen ejemplo se encuentra en el ejido Koiyaki: los habitantes dependen tanto del ecoturismo que cualquier disminución de la demanda, por ejemplo de las llegadas de ecoturistas, tendría efectos adversos en cuanto a su sobrevivencia (conclusión basada principalmente en las encuestas). En caso de haber una reducción significativa de la actividad ecoturística, casi todos los encuestados indicaron que volverían a sus antiguas actividades económicas (la mayoría de las cuales no son compatibles con los objetivos de conservación, por ejemplo, la agricultura masiva). 
En Adolfo López Mateos, la comunidad se ha quedado sin ningún tipo de diversidad económica que actúe como una alternativa si disminuye el ecoturismo. Los resultados del presente estudio concuerdan con los planteamientos de Brasileiro y Tovar (2017) que destacan las limitaciones o desventajas de la dependencia excesiva del turismo. Según ellos, la economía local queda vulnerable durante temporadas bajas. Además de la dependencia excesiva, la mayoría de los encuestados en Koiyaki y Adolfo López Mateos considera que la comunidad ha perdido el control sobre los precios de bienes y servicios esenciales, y argumenta que el ecoturismo ha causado un enorme aumento de los precios.

En Koiyaki, los miembros de la comunidad no pueden permitirse el lujo de pagar precios exorbitantes que se cobran en los centros comerciales locales por productos básicos como agua mineral. Brasileiro y Tovar (2017) abordan esta cuestión de forma detallada, y señalan que la excesiva dependencia del turismo puede llevar a la comunidad a una especie de desastre económico, en el cual sus miembros ya no pueden comprar comodidades y servicios básicos; con el tiempo se convierten en comunidades pobres que sirven a una industria rica. Es importante aclarar que ninguna de las dos comunidades (Koiyaki y Adolfo López Mateos) ha llegado a este estado (y, probablemente, nunca lo harán), sin embargo, se debe reconocer que sus economías se han transformado de manera significativa, llegando a ser bastante dependientes de la industria ecoturística.

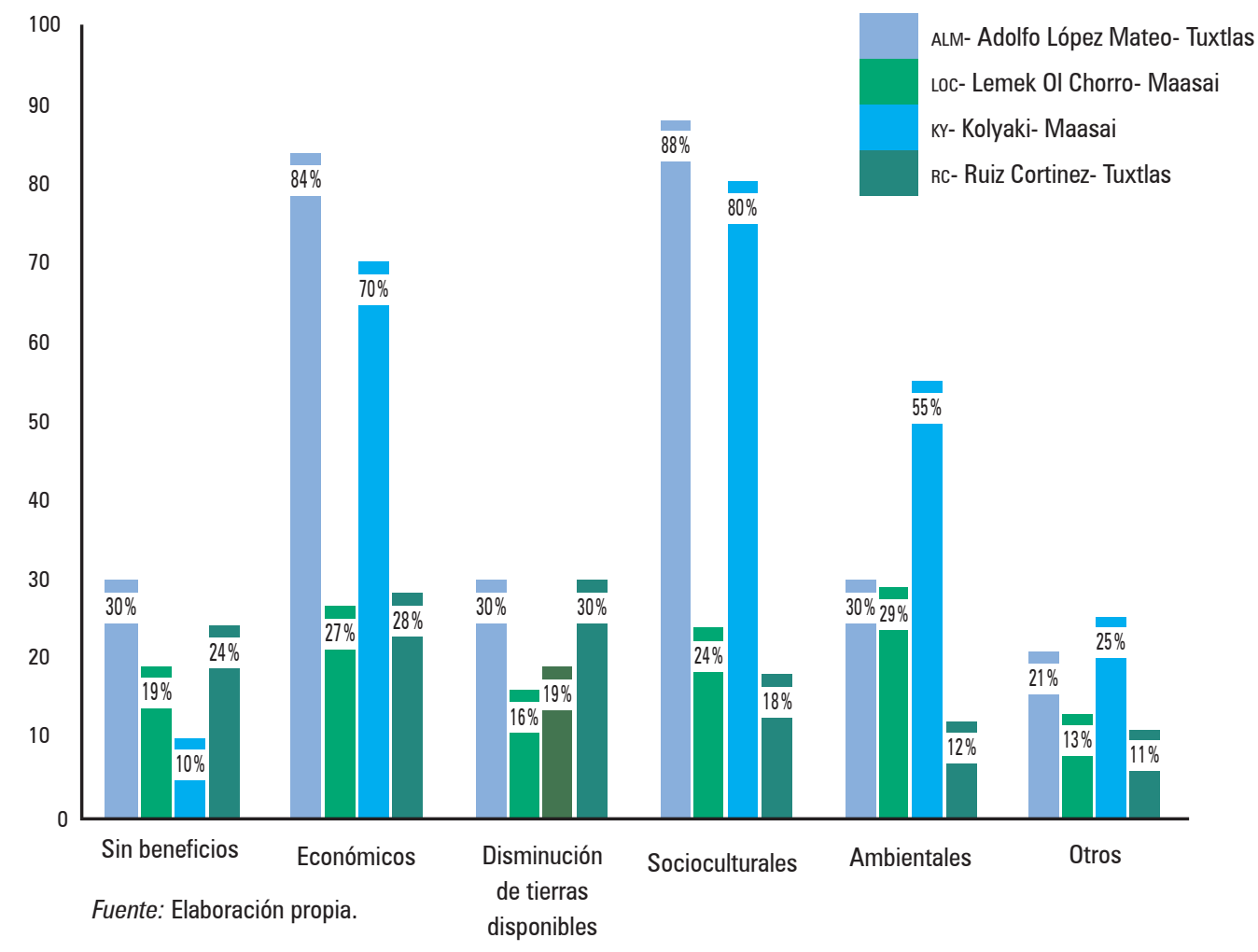

Figura 3. Los impactos del ecoturismo en los cuatro grupos ejidales 
Con relación a los impactos generales del ecoturismo, la figura 3 ilustra claramente que el aumento de la actividad ecoturística ha originado diversos efectos negativos, sobre todo en las comunidades donde la actividad se realiza a una escala más amplia. Los encuestados de Lemek/OI Chorro y Ruiz Cortines refieren menos impactos negativos (por ejemplo, consideran que su participación en el ecoturismo es mínima y no les permite generar beneficios), mientras los de Koiyaki y Adolfo López Mateos indican los diversos impactos negativos del ecoturismo: un aumento de los problemas ambientales como la contaminación, e impactos socioculturales como la pérdida de culturas indígenas y desintegración general de la comunidad (en el caso de Koiyaki).

Existen diversos estudios que examinan los impactos del ecoturismo. Poudel y Nyaupane (2017) exploran impactos ambientales causados por el comportamiento de los turistas, como la contaminación. Por otro lado, Karst (2017) aborda los efectos socioeconómicos y culturales, por ejemplo, escasez de beneficios para la comunidad local, conflictos entre sus miembros cuando tratan de competir por los beneficios del ecoturismo, vulnerabilidad de la economía local cuando se depende demasiado del ecoturismo, desintegración de las comunidades y familias debido a los conflictos relacionados con el uso de los recursos y distribución de beneficios. Asimismo, Honey (2008) presenta diversos puntos de vista, con ejemplos empíricos generados de estudios comparativos que abarcan proyectos de ecoturismo en diferentes países, y es común entre sus resultados la afirmación de que el ecoturismo produce impactos negativos, los cuales se deben controlar para que la actividad logre objetivos de sustentabilidad.

\section{Conclusiones}

La presente investigación consolida el acervo de conocimientos sobre el ecoturismo y la conservación; se generaron aportaciones teóricas y prácticas para conciliar la conservación con el desarrollo local. Siendo el estudio de caso comparativo, que abarcó cuatro casos en dos países, se tiene importancia metodológica; a través de la comparación se logró esclarecer las similitudes y diferencias entre las cuatro comunidades, y las condiciones en que ocurren, lo que permite comprender mejor cómo se relaciona el ecoturismo y la conservación bajo las circunstancias contextuales de cada una.

Se concluye que el ecoturismo puede engendrar importantes beneficios económicos para las comunidades locales e incentivar su participación en iniciativas de conservación, evitando prácticas destructivas, como la tala de árboles, la cacería o actividades que implican uso intensivo de los recursos naturales, como la agricultura. Los resultados demuestran una asociación significativa entre empleo en la actividad ecoturística y las prácticas de conservación; aún así, la relación entre el ecoturismo y las actitudes hacia la conservación parece ser estadísticamente insignificante. Tambien se concluye que los incentivos económicos directos no son los únicos factores que influyen en las actitudes y prácticas de conservación; los beneficios indirectos del ecoturismo, como la interacción e intercambio de ideas con ecoturistas, la participación local, la distribución de ingresos y la formación 
y capacitación, mostraron asociaciones o tendencias claras y positivas con las actitudes hacia la conservación, por lo tanto, se reconoce su importancia como factores determinantes. Es decir, los beneficios del ecoturismo van más allá del ingreso económico directo. Se deben contemplar estrategias para asegurar ambos beneficios (directos e indirectos).

El análisis del ecoturismo como factor predictor de las actitudes y prácticas de conservación comprueba las tres hipótesis planteadas al inicio del estudio: 1. Incentivos económicos por sí solos no son suficientes para que las comunidades locales abandonen sus prácticas económicas tradicionales a favor de la conservación. Otros factores, como las leyes y restricciones, la distribución de los beneficios, el involucramiento y empoderamiento, motivan e influyen en las actitudes y prácticas hacia la conservación; 2. Una mayor participación de las comunidades locales en las actividades del ecoturismo y la distribución equitativa de los costos y beneficios pueden generar actitudes y prácticas positivas que apoyan a la conservación; y 3 . El ecoturismo puede influir negativamente en la conservación como resultado de acciones involuntarias o equivocadas.

Con base en los resultados obtenidos en esta investigación, se puede concluir que no existe un nivel de la actividad ecoturística ideal que no se considera excesivo, y ofrece máximos beneficios con mínimos impactos. Así pues, el éxito o fracaso de la actividad ecoturística puede variar en diferentes casos y depende del contexto local, además de los factores sociodemográficos, como la cultura.

Por otro lado, se distingue un caso de otro por los enfoques y estrategias empleados principalmente por los operadores ecoturísticos para integrar la conservación y el desarroIlo de las comunidades. Cuando el ecoturismo ocupa un papel importante en la economía local, y ofrece beneficios sin perjudicar los recursos naturales que son la base económica de la comunidad, existen posibilidades de integrar la conservación y el desarrollo socioeconómico. Sin embargo, se recomiendan estudios más críticos y profundos para evaluar las relaciones de poder entre los diferentes actores y las comunidades. El ecoturismo puede generar impactos negativos cuando no se maneja de manera apropiada. Se debería ir más allá de la suposición de que el ecoturismo es una alternativa económica absolutamente segura que contribuye al bienestar de los recursos naturales y de las comunidades locales; en contextos ideales, lo afirmado sería cierto. 


\section{Fuentes CONSULtadas}

Adams, J. S. y McShane, T. O. (1992). The Myth of Wild Africa: Conservation without Illusion. Berkely y Los Ángeles: University of California Press.

Adams, W. M. y Hutton, J. (2007). People, parks and poverty: Political ecology and biodiversity conservation. Conservation and Society, 5(2), 147-183.

Agüera, F. O. y Morales, P. C. (2015). Ecoturismo y desarrollo sostenible. Un estudio de caso en comunidades rurales de República Dominicana. PASOS. Revista de Turismo y Patrimonio Cultural, 13(6), 1425-1435.

Barriga, A. M. (2015). La contradicción del turismo en la conservación y el desarrollo en Galápagos-Ecuador. Estudios y Perspectivas en Turismo, 24(2), 399-413.

Barriga, A. M. (2017). Percepciones de la gestión del turismo en dos reservas de biosfera ecuatorianas: Galápagos y Sumaco. Investigaciones Geográficas, Boletín del Instituto de Geografía, 93, 110-125.

Bello, F. G., Lovelock, B. y Carr, N. (2017). Constraints of community participation in protected area-based tourism planning: The case of Malawi. Journal of Ecotourism, 16(2), 131-151.

Björk, P. (2007). Definition paradoxes: From concept to definition. En J. E. Higham (ed.), Critical Issues in Ecotourism: Understanding a Complex Phenomenon (pp. 23-45). Oxford: Butterworth-Heinemann.

Brasileiro, M. S. y Tovar, L. S. (2017). Actividad turística y empleo. Una realidad entendidad desde los actores. Paradigma, 24(2), 87-111.

Brenner, L. y Job, H. (2012). Challenges to actor-oriented environmental governance: Examples from three Mexican biosphere reserves. Tijdschrift voor economische en sociale geografie, 103(1), 1-19.

Butler, R. (1980). The concept of a tourist area cycle of evolution. Implications for management of resources. Canadian Geographer, 24(1), 5-12.

Cardoso-Jiménez, C. (2006). Turismo sostenible: una revisión conceptual aplicada. El Periplo Sustentable, 11, 5-21.

Castillo, S. A., Baltazar, E. B., Lugo, E. E. y Piñera, E. N. (2016). Procesos organizativos, turismo y conservación en la reserva de la biósfera Ría Lagartos, Yucatán. Estudios Sociales, 25(47), 164-187.

Ghimire, K. B. (1994). Parks and people: Livelihood issues in national parks management in Thailand and Madagascar. Development and Change, 25(1), 195-229.

Gómez, N. S. (2011). La planificación de los destinos turísticos mexicanos: una receta mil veces vanagloriada. Renovación y restructuración de destinos turísticos consolidados del litoral, Bloque temático 1: Principios y fundamentos de los procesos de renovación y reestructuración en el marco del ciclo de vida de los destinos turísticos (pp. 1-21). Alicante: Universidad de Alicante.

Guerrero Rodríguez, R. (2010). Ecoturismo mexicano: la promesa, la realidad y el futuro. Un análisis situacional mediante estudios de caso. El Periplo Sustentable, 18, 37-67.

Higham, J. (2007). Ecotourism: Competing and conflicting schools of thought. En J. Higham, Critical Issues in Ecotourism: Understanding a Complex Phenomenon (pp. 20-40). Oxford: Butterworth-Heinemann. 
Honey, M. (2002). Ecotourism and Certification: Setting Standards in Practice. Washington, D. C.: Island Press.

Honey, M. (2008). Ecotourism and Sustainable Development: Who Owns Paradise? Washington, D. C.: Island Press.

Hvenegaard, G. (1994). Ecotourism: A status report and conceptual framework. Journal of Tourism Studies, 5(2), 24-35.

Instituto Nacional de Estadística y Geografía. (2010). Censo de Población y Vivienda 2010. México: Autor.

International Union for the Conservation of Nature. (1994). Guidelines for Protected Area Management Categories. Gland y Cambridge, Reino Unido: International Union for the Conservation of Nature-World Monitoring Conservation Centre.

Karst, H. (2017). "This is a holy place of Ama Jomo": Buen vivir, indigenous voices and ecotourism development in a protected area of Bhutan. Journal of Sustainable Tourism, 25(6), 746-762.

Kenya National Bureau of Statitics. (2012). Statistical releases. Nairobi: Government of Kenya. Recuperado de https://www.knbs.or.ke/data-releases /

Laven, D. N., Wall-Reinius, S. y Fredman, P. (2015). New challenges for managing sustainable tourism in protected areas: An exploratory study of the European Landscape Convention in Sweden. Society \& Natural Resources, 28(10), 1126-1143.

Lawton, L. J. y Weaver, D. B. (2015). Using residents' perceptions research to inform planning and management for sustainable tourism: A study of the Gold Coast Schoolies Week, a contentious tourism event. Journal of Sustainable Tourism, 23(5), 660-682.

Magio, K. O., Velarde, M. V., Santillán, M. N. y Ríos, G. (2013). Ecotourism in developing countries: A critical analysis of the promise, the reality and the future. Journal of Emerging Trends in Economics and Management Sciences (JETEMS), 4(5), 481-486.

Marín, G., García, A. y Daltabuit, M. (coords.). (2012). Turismo, globalización y sociedades locales en la península de Yucatán, México (PASOS edita, 7). Tenerife: PASOS. Revista de Turismo y Patrimonio Cultural.

Morse, J. M. (2003). Principles of mixed methods and multimethod research design. En A. Tashakkori y C. Teddlie (eds.), Handbook of Mixed Methods in Social and Behavioral Research (pp. 189-208). Thousand Oaks: Sage.

Palomo, I., Montes, C., Martín-López, B., González, J. A., García-Llorente, M., Alcorlo, P. y Mora, M. R. G. (2014). Incorporating the social-ecological approach in protected areas in the Anthropocene. BioScience, 64(3), 181-191.

Patton, M. Q. (1990). Qualitative Evaluation and Research Methods (2a ed.). Newbury Park: Sage.

Picard, D. (2015). Making ecotourism sustainable: Refocusing on economic viability. Lessons learnt from the "Regional strategic action plan for coastal ecotourism development in the South Western Indian Ocean”. Journal of Sustainable Tourism, 23(6), 819-837.

Poudel, S. y Nyaupane, G. P. (2017). Understanding environmentally responsible behaviour of ecotourists: The reasoned action approach. Tourism Planning \& Development, 14(3), 337-352.

Primack, R. B. y Ros, J. (2002). Introducción a la biología de la conservación. Barcelona: Ariel. 
Siewe, S., Vadjunec, J. M. y Caniglia, B. (2017). The politics of land use in the Korup National Park. Land, 6(1), 7.

Toledo, V. M. (2005). Repensar la conservación: ¿Áreas naturales protegidas o estrategia biorregional? Gaceta Ecológica, 77, 67-83.

Unesco. (1995). Reservas de la biósfera: La estrategia de Sevilla y el marco estatutario de la red mundial. París: Autor.

Unesco. (2005). Biosphere Reserves Benefits and Opportunities, Man and the Biosphere Programme. Recuperado de http://portal.unesco.org [2016, 25 de noviembre].

Vargas del Río, D. y Brenner, L. (2013). Ecoturismo comunitario y conservación ambiental: la experiencia de La Ventanilla, Oaxaca, México. Estudios Sociales, 21(41), 31-63.

Weaver, D. (1998). Ecotourism in the Less Developed World. Wallingford: Cab International.

Weaver, D. (2006). Sustainable Tourism: Theory and Practice. Oxford: Elsevier Butterworth-Heinemann. 
